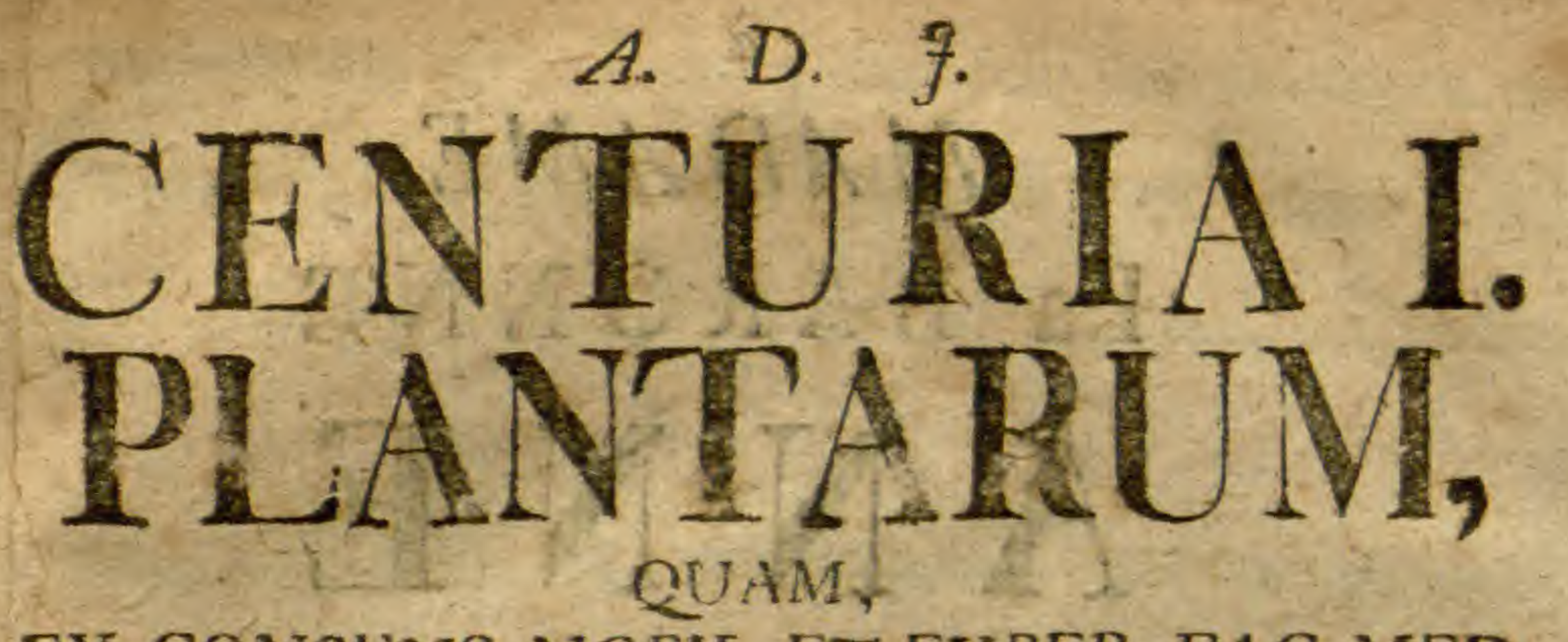

EX.CONSENS. NOBHLET EXPER. FAG.MED. IN REGIA A CADEMIA UPSALIENSI,

\title{
SUb PRIESIDIO,
}

VIRI NOBIDISSIMI AT QUE CELEBERTIMI
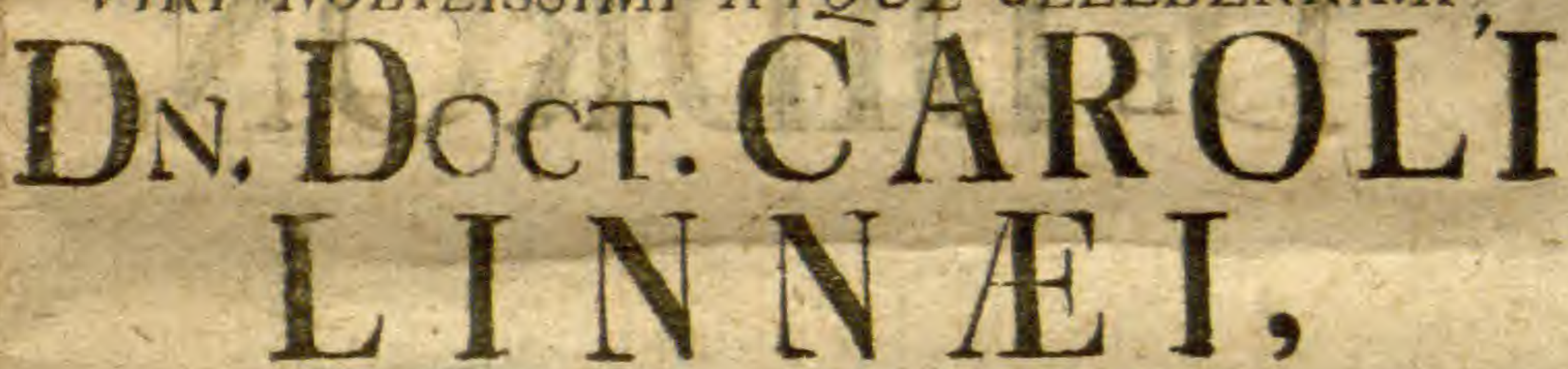

S: $\mathbb{E}$ S:AE M:TIS ARCHIATRI,

Med. et Botan. PRUFESS. Reg. et Ord.

Acad. Imp. N. C. Lond, Monspel. Berol. Tolos.

FLORENT, STOGKHOLM, ET UPSAL. SOCII, Net not

Equitis Aurati de Stella Polari, ORBI BOTANICO FXAMINANDAM SISTIT,

\section{ABRAHAM. D. JUSLENIUS,}

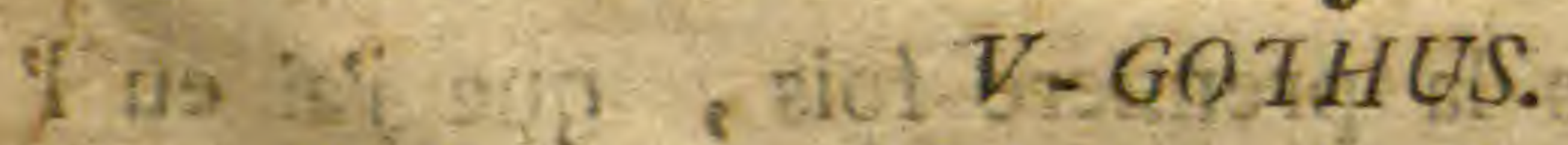

IN AUDIT, GUSTAV. DIE XIX. FEBRUAR.

ANNI MDCCLV.

in H. A, M. S.

UPSAL1/E, Exc, L. M. HO̊JER, Reg. Acad.Typogr,

MU. BOR QANOD $\quad \beta$ 1911 


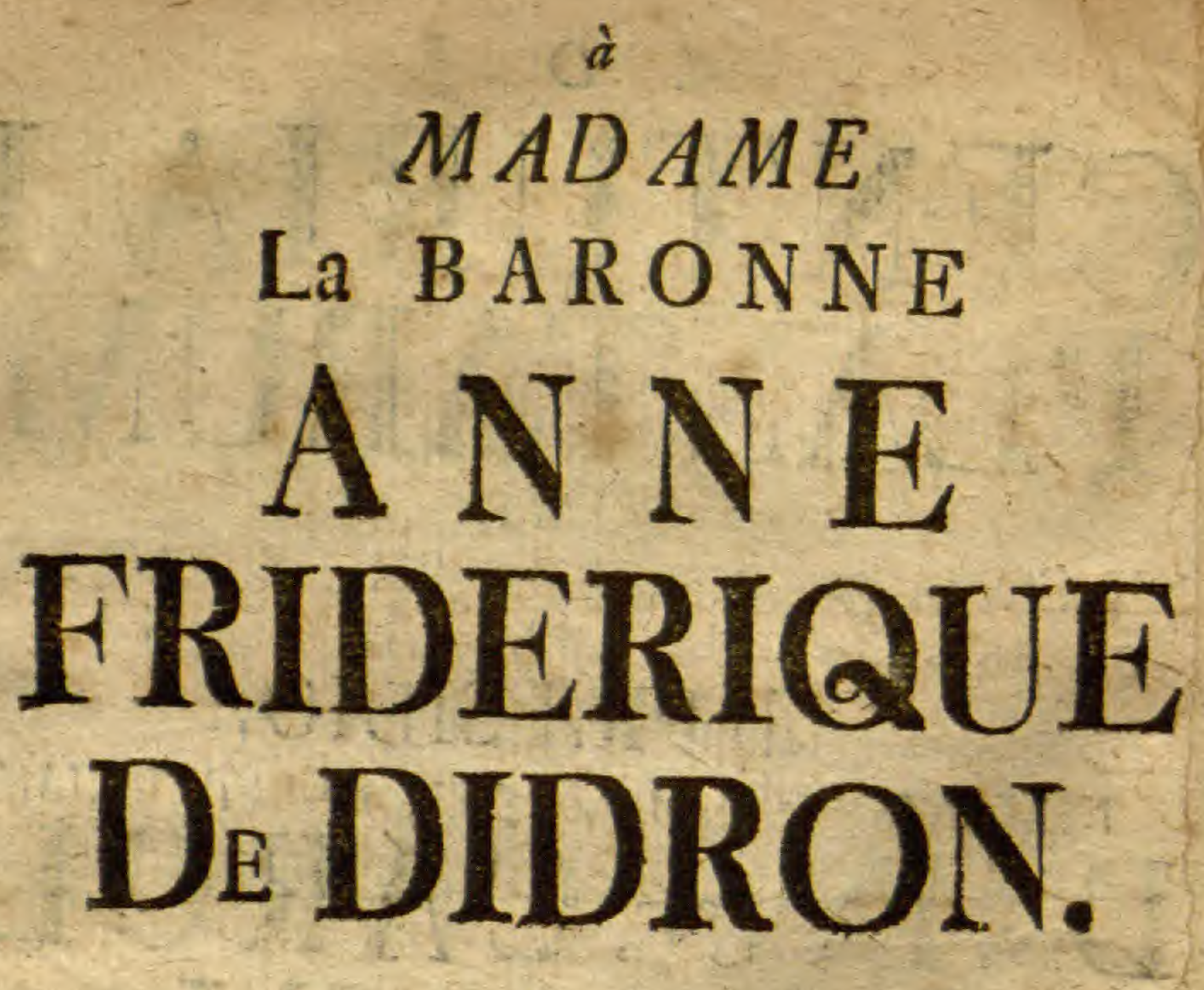

1 fe prefente une occafion fort convenable
faire voir au public les obligations, que je Vous ai de la grace, que Vous $\mathrm{m}^{2}$ avez te moignée depuis la premiere tois, que j’ai eu 1 honneur de Vous etre conna. J'ai le bonheur main 
maintenant de Vous prefenter ces premiers travaux academiques. Il eft jufte, qu' apres avoir été comblé de tant de bontés, je taffe connoitre à tout le monde la gloire, que Vous Vous faites, MADAME, de me favorifer de Vos bienfaits, \& combien i’en fuis penetré de recontoifance. Il ne me refte, MADAME, qu' à prier Le tout Puiflant qu' il Vous conferve en fanté, \& Vous faffe jouir de toutes les profperites defiderables, tant pour Sa Gloire, que pour celle de toute Votre illuftre Famille. Ce font des voeux tres ardens, qui emanent d' un coeur qui Vous fera devorié jusques au dernier de mes foupirs. Je fuis avec bien de refpeet,

\section{$M A D A M E$}

- Votre très bumble E très obeiffant Serviteur, ABRAHAM JUSLENIUS. 


\section{$\operatorname{Nin} B R O R$.}

Fru Floras kärlek elde opp

Din vördnad, för Den henne

$$
\text { pryder, }
$$

MinBror,fâ grön/kas vift mitt hopp, Att ön kan din blid lycka lyder,

Tils Floras multna drägt firt kläder Ditt ftoft, och Himlen frälengläder. MARG, JUSLENIA. 

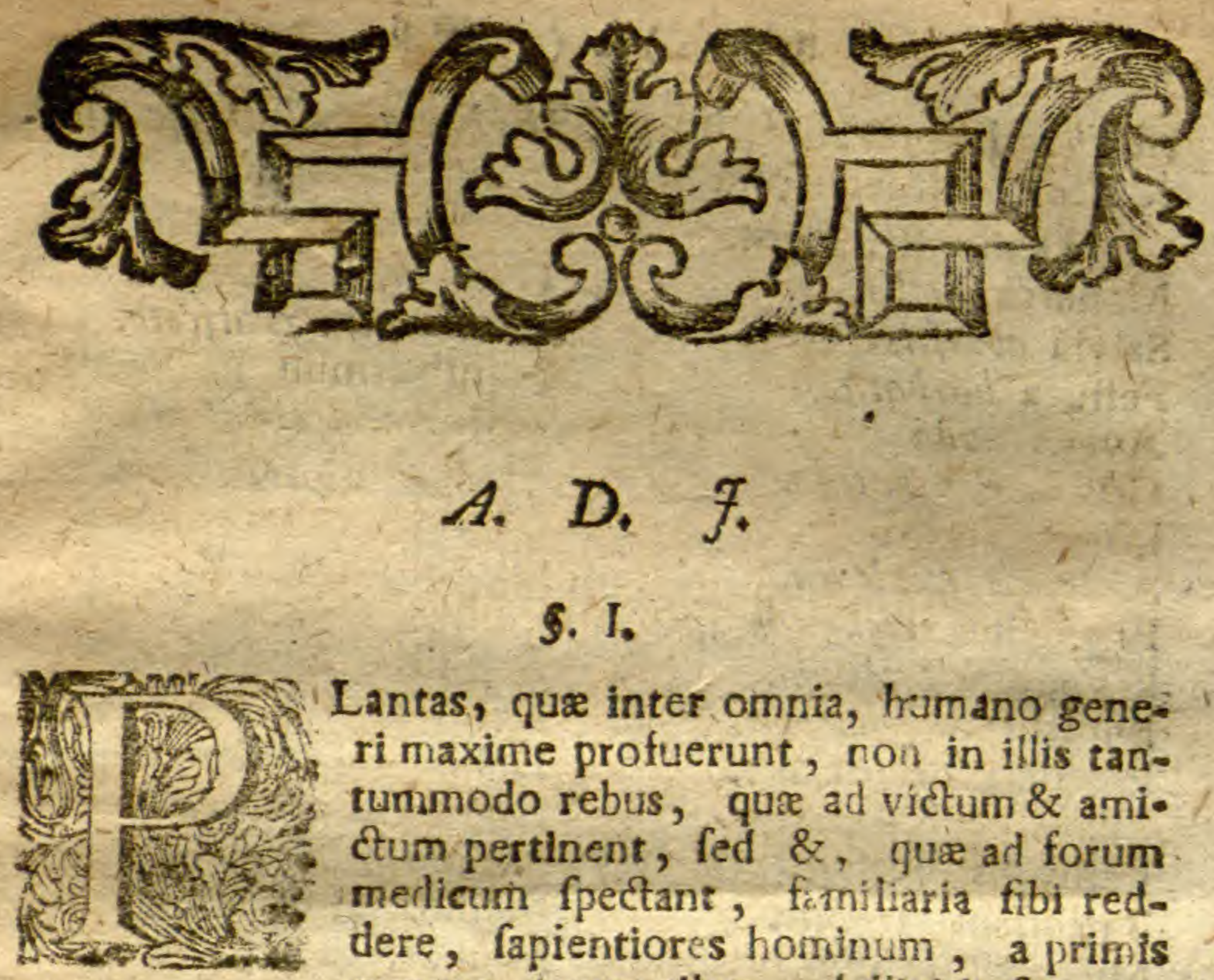

Lantas, quæ inter omnia, humano generi maxime profuerunt, non in illis tantunmodo rebus, qua ad víctum \& amictum pertinent, fed \&, quæ ad forum medicum fpectant, farniliaria fibi reddere, fapientiores hominum, a primis quoque temporibus, folliciti fuerunt. Medicis, præ ceteris, maximam certe debemus gratiam, quod cognitionem plantarum in formam difciplinz redegerint, noftrumque eft fcientiam hanc magis magisque excolere. Quantun luminis, nobiliflimæ huic arti noftris temporibus acceffit, omnibus notum eft, adeo ut nullam plantam accurate defcriptam \& cognitam agnofcamus, priusquam illam ad proprium genus referre, atque adxquata differentia fpecifica ab aliis diltingvere poffmus. Nobiliffimus Dominus PRASES Joctrinam hane in fuis Speciebus Plantarum valde neceffariis optime perpolivit, in quibus omnes illas plantas, qure tunc temporis quoad fructum \& reliquas partes fatis perfpectre fuerunt, rite delineavit. Quot vero plantæ, in hancce Flora f́cenam, accuratis \& concinnis deferiptionibus ornatæ poftea prodiere, in duobus Speciminibus Academicis, Demonftra- 
tionibus Plantarum fcilicet \& Herbario Amboihenfe videre licet, ubi fequentia ante oculum pontntur.

Demonitr. Plant.

Monarda mollis,

Salvia agreftis.

Feftuca barbata.

Avena nuda.

Crucianella patula.

Datura ferox.

Afclepias exaltina.

Er yngium tricufpidatum. .

Erynglum trifidum.

Eupleurum femicompofitum?.

Eryfimun renanduin.

Malva parvifiora.

I athyrus annuzs.

Trifoliom retufum.

Micropus exedus.
Herbar. Amb.

Piper decumanum.

Convallaria fruticola.

Menifpermum flavum.

Adenanthera folcata.

Rhizophora cefeolaris.

Rhizophora conniculata.

Garcinia celibiea.

Pfidium Cujavzes.

Myrtus lesseadsntra.

Dotichos tetragunalobus.

Dolichos pruriens.

"Hernandia ovizera.

Ricinus Mappa.

Ricinus Tanarius.

Momordica trifolia.

Ophiogloffum pendulum.

\section{E. II.}

Ex Speciebus Plantarum unicứque patet, quot plante peregrinæ \& antea incognitæe ex indefeflo lsbore nofiratium, ut exteros reticeam, utpore Celeberrimi Aboenfum Profefforis KALM, OSBECK, LOFLING, dudum innotuerunt: Hifce accedunt muitre, exque exoticæ, qua uftimis hifce annis ex intuftria Virorum, de te herbaria opiime meritorum; LSELING, b. m. Doctoris HASSEL QUIST, SAUVAGES, AYMEN, MONNiER, reliquorum, invente \& accurate defcripta funt. Unicuique igitur, qui nobil fimam Kanc artem pretio habet, illam tam immobilibus fuperftructam fundamentis augere in junctum efle crediderim. Ego equidem, qui per aliquod temporis fpatium ftudiorum gratia ad hanc amceniflimain Mufa- 
Mufarum fectem commoratus fum, \& qui jucundifimam hanc doctrinam, primis tantum, ut ajunt, labris tetigi, illam tanto amplexus furm amore, adeo ut plantam minus frequentem \& rariffime obviam vel ut gemman quandam pretiofam aftimaverim, mihigue magis gratum fuerit, fi illam rite explicare \& accurate defcribere valuerim, quam fi vel difficillimum problema refolviffem, \& quoniam mihi copia fuit, plurimas plantas exoticas atque nuperrime detectas; apud Nobiliffimum Doninum PR ASIDEM videndi, ipfurn exoravi, ut mihi poteftas daretur, ex illis centuriam eligend, eamque ut primum tirocinium botanicorum difquifitioni fubmittend, quam L. B. xqui bonique confulere dignetur, qua par eft animi veneratione, humillimus oro.

1. VER ONICA (montana) racemis lateralibus paucifloris, calycibus hirfutis, follis ovatis rugofis crenatis p tiolatis, caule debili. $t$

Veronica procumbens. Riv. irr. 92. Hall. gett. 2, p. 235. defcr.

Chamrdrys fpurie adfinis rotundifolia fcutellata. Baub. pin. 249.

Alyffon diofcoridis montanum. Col. ecpbr. 1. p. 286. t. 288.

Habitat in montanis Mattefii Italix \& Greifmari Get: manias umbrofis.

2. ZIZIPHORA (bi panica) foliis ovatis, floribus racemofo-fpicatis, bracteis obovatis nervolis acutis.

Halitat in Hifpania. Laefl. 44\%.

Defer. Caulis brachiatus. Folia ovata, petiolata, vix crenata. Spice laxe, racernos caulemque terminantes, oblongæ: Bracteis folio majoribus, obovatis, nervofis, acutis, integerrimis, ciliatts. Flores terni intra fingulam Bracteam, longitudine bractex, Calyzes 
cylindriel, inferne craffiores, feffies, hifpidi. Corolte limbe parve. Stanina duo.

3. IRIS (pyrrinaica) corollis barbatis? foliis repandis.

Iris humilis pyrenaica, foliis repandis, e luteo virefcentibus. Tournet. inft. $36 r$.

$\beta$ Iris humilis pyrenaica, follis repandis virefcentibus cum lineis ceruleis. Towrnel.infl. 361 .

Habitat in Pyrenæis.

4. SCHOENUS (capenfis) culmo tereti Iamofilimo vaginato, fpicis ovatis nudis.

Canna capitis bonæ Ipei, fpicis juliformibus. Scbeuchz. gram. 352.

Juncus e capite bonæ fpei, paniculis fufcis juliformibus. Petivg gz t.7.t.15. Muf. $4^{2} 4$.

Hnbitat ad Caput Bonx Spel.

5. LYGEUM (Spartam.)

Lygeum fpartum. Locfling.

Spartum herba alterum. Clu/.bift.2. p.220.f. 2.

Gramen fparteum fecundum, paniculabrevi, folliculo inclufa. Baub. pin.5.

Habitat in Hifpania.

6. PHALARIS (nquatica) paritcula fpiciformi ovatooblonga, glumis carinatis lanceolatis.

Gramen typhinum phalaroides majus bulbofum aquaticum. Barr. rar. I200.t. 700.f.I.

Habitat in 2 Egypro of ad Tyberin.

Defcr. Culmus arundinaceus, foliis folidiufculis. E vaginafolit fupremi fubventricofa Spica ovato-oblonga iglabra, vel potius panicula ardta. Glume lanceo. late, glabre, serinatæ, utrinque nervo exaratæ.

7. PHALARIS (bulbofa) fpica cylindrica, glumis carinatis.

Phalajis bulbofa, albo femine. Słberua gram. 53.

Habitat in oriente.

Defer. 


\section{eeg $10($ cos}

Dejer. Spica pollices duos longa: Glumis carinatis, ovatis, minoribus, arcte in fpicam congeftis.

8. POA (rigida) panicula lanceolata fubramofa, floribus alternis fecundis, Royen. Lugd-bat. 62. Gutt, Ramp. 6?. Dalibard.paril.30.

Granen filiceum rigidiufculum. Vaill.pariJ.p.92. t. 18.f.4. Habitat in Gallia, Anglia. ๑.

Confer. Gramen loliaceum murorum cluriufculum, fpica erecta rigida. Moril. bift. 3. p. 182.1.8.t.2\% . . Gramen panicula -sultiplicl. Baub. pin 3.prodr.6. Granen exile duriufculum in muris \& aridis proventens. Raj. angl. 410.

9. BROMUS (madritenfis) panicula rariore erecta, fpi. culis lineribus: intermedilis geminis.

Bromus panieula fpicata rariflima, fpieulis linearibus alternis : pedicellis increffaris. Lefling.

Gramen bromoides pumilum, locultis erectis majoribus ariftatis. Scbeuchz. gram 260.

Bromos fterilis, erecta panicula, major. Barr. icon. 76. n.r. Habitat in Hifpania.

Defor. Culmi bipedales, leves, geniculis tumidis. Folia latiufcula, mollia. Panicula laxa, rarior. Spicula rectr, lineares, erectiufcule, terna, fapius proprilis pedunculis, \& quartus infuper peduneulus fepius diftachyos. Arifte longa, erectr.

10. BROMUS (rubens) panicula fafciculata, fpiculis fubfeffilibus villofis; ariftis erectis.

Gramen panicula molli rubente. Barb bif. 2. p. 404?

Habitat in Hifpania. Loefling.

- Defrr. Spicule numerofe, oblongx, villofo-fcabra, glumis fubulatis, longe ariftatis, in fafciculun ovarum congeftre, fubfeffiles. Gluza fupra ariftan acuta eft; gluna exterior ciliata:

11. BRO. 
11. BROMUS (jeoperizs) pani ula fafciculata, ficulis fubfeff bilibus glabris, ariltis patuls.

Hobitat in Hipa ia.

Dejer. Pamicula ovata ex piculis fubf filibus, ovatooblongis, glabris; arjftis non erectis, fed extrorfum verfis; Gluma fuypra ariftam obrufa eft.

12. STIPA (tenacilfina) arintis bafi pilofis, panicula (picata, foliis tilformibus. Loefling.

Gramen fparteum I, panicula comofa Baub. pin.s.

S artum herba Plinii. C.lufir b:ft.2. p. 220.

Habitat in Hifpanix collibus fajulofis.

13. TRITICUM (juncetum) calyctbus trúncatis quinquefloris, folis involutis.

Gramen, tripiici fpica mutice fimili, anguftifolium Baub. pin. 7. theatr. 132.prodr. 18.t. 17. Scbeucliz. gram. 7.

Habitat in Helvetia, Oriente. 4

14. ELYMUS (philadelphirus) fpica pendula; fpiculis fexflor is : inferioribus ternatis, fuperiuribus binatis.

Habitat in Penfylvania, Kalim. 24

De/cr. Habitus \& tota ftructura fimillima canadenfi, fed Spica in hac omnino apice nutat ultra bafin; at in itla fpica parum obliqua perfintit, Spicula in hac fexflore, in illa quadriffore, fed inorimis 1 icule, \& prectpue intermedia, in hac latus planum uti triticum culno ob-

- vertit. in illa \& reliquis lafus angulaturn fcapo obver-

tit. Hec ariftas minus rectas, ext pirum flexuofes, etjam dum floret, illa veru rectas habet; hoe fpicas magis villofas, quam illa gerit.

15. SCABIOSA (graminifolia) corollulis quinquefidis; foliis lineari-lanceolatis integerrin is, caule berbaceo. Scabiofa argentea anguftifolia. Borib. pirs. 2>0. prodr. 127. f. 127 .

Scabiofa graminea argèntea, Baub. bill.3.p.I2. 


\section{1 es $) 0($}

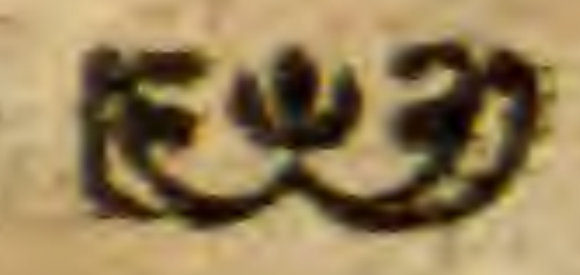

Afterocephalus argenteus graminifulius, flore cxrulea. Vaill. paril. 248 .

Hobitat in alpibus Helveticis. 4

16. CERINTHE (orientalis) foliis lanceolatis hifpidis,

feminibus nृuaternts diftinctis, fructibus pendulis.

Symohytun orientale, echil folio minori. Tourn.cor. 6?

Havitat in Tigypto b.m D. Hoflelquift.

Defcr. Comles teretes, hifpidi. Folsa aiterna, feffilia, integerima, hilpida pilis viridibus aut albicantibus. $R a-$ mas termin sus duplici racemo: Bracteis lanceolatis, anguftionbus, hifjidis. Flores pedunculati; Calyis foliola e cordara kgura in linearem transeunt. Corolla quinqu fila fegmentis fubulatls, acutis, patulis; Jtamina fubulata, longitudine corolla. Differt ergo a Cerinthe echioide; qiuod non pilis flavefcet; quod corolla non obrufa; quodque calyx fruckus non erectus, nec foliolis lineari-lanceolatis.

17. MIRABLIS (eitorata) caule dichotomo, corollis limbo parentibus, ftaminibus ptftilo brevioribus.

Defer. Similis Mirabili Jalappe, fed Folia magis cordata \& magis acurrinata. Coulis \& rami dichoremi ; Flo. ribus e dichotomia. Corolle limbo patente nec erecto; Staminibus piftillo fenfrim brevioribus, nec longirudine equalibus; Corollis odoratis, tubo corolloe fubpilofo.

18. MIRABILIS (longiflora) foliis villofis, corollis folio Iongioribus.

Mirabilis caule follisque villofis \& glutinofis, tubo fioris pedali. Monnier.

Defer. Planta tota villofa, vifeofa. Folia fumma feffilia, cordata. Flores terminales, fafciculati. Corolle tubus filiformis, femipedalis, villofus, laxus, limbo albo, tubo 
tubo breviore. Stamina \& piftilla intra faucem corollex : Stigma valde muricatum.

Ob/. Diftinguatur itaque Mirabilis ( Falappan) foliis corollisque glabris, floribus congefts terminalibus.

19. SOLANUM (pimpinellifolium) caule inermi herbaceo, follis pinnat's integerrimis, racemis fimplicibus. Habitnt in Peru. ()

Delcr. Habirus \& ftatura \& omnia Solani Lycoperfici. Fructus frmiles varictati $\beta$, at differt a 9. Sp. PI. omnino, 1.) cante lavi, nec ullis pilis, neque in pedunctlis, adfperfis uti illa. 2.) Folvis glabris, integris, cordatis, nec ullo modo incilis, aut dentatis, utj illa. Creterum forte orte ab illa, uti toka ftructura convenit. Pedunculi ftructura ut in 8.9.10. Sp. $\mathrm{Pl}$. pecticellis etjam geniculatis, racemonudo, ut $\$ 9$ ibid., nec follofo út ro.

20. PHYLICA (radima) foliis linearibus tiquetris, Iparfis caule pilofo, bracteis apice coloratis glabrisque. Chryfanthemum ericoides coronatum capitis bonze fpei. Breyn. cent. 165. t.82.

Habitat ad Caput Bone Spei. B

Defcr. Frutex ramis tenuibus, pilis longis raris adfperfis. Folia fparfa, linearia, triquetra, fere canaliculata, acuta, raris pilis frepius adfperfa. Flores aggregati, fesfiles, folitarii, laterales, cirictir radio albo ebrueteis foliaceis, apice niveis, glabris. Flofcult foffiles, radio breviores, quinquefidi, cum llamine \& Iquamula petali hitra fingulam laciniam corolla; iylus fimplex.

2r. HFRNIARIA (fruticoja) caulibus fruticolis, floribus quadrifidis.

Herniaria fruticola, viticulis lignofis. Iotr'n. inf. 507. Halitat in Hifpania. $\hbar$

Defer. Calyx teiraphyllus. Stamina quatuor tertilia, \&z quatuor fterilia loco petalornin. Zeffling. 
22. BUPLEURUM (fruticefcens) frutefcens, foliis linearibus, involucro univerfali partialibusque. Laefling. Bupleurum fruticans anguftifolium hifpanicum. Barr. rar. $623 . t, 1255$

Bupleurum hifpanicum arborefcens, gramineo folio. Tourn. inft. 310.

Habitat in Hirpanix collibus altis. $\hbar$

23. SELINUM (Monnieri) umbella conferta, involuero univerfali redexo, feminibus quinque coftis membranaceis.

Athufa f. Cicuta minor hirfuta, femine parvo. Monnier. Habitot in Gallia auftrali.

Defcr. Planta Ethufam refert, fed folia tenerius tripinnata. Coulis pedalis, angulatus, Ixvis. Rami multi, alterni, fubdiviti, caule altiores. Folia tripinnata, la. via, legmentis fublinearibus, obtufiufculis, planis, lae. vibus, non vero nitidis. Pedunculi univerfales membranacei, amplectentes ramos. Uimbella conferta. Involucrum univerfale fimplex, fetaceum, reflexum, umbella brevius ; involucra partialia fetacea, longitu* dine umbellulx. Flores albi, pentapetali : petalis equalibus, cordatis, acumine intermedia incurvato. Stamiha alba. Styli duo, albi, filiformes, perfintentes. Semina parva , ovata, quinquecoftata, membranacea, Iongitudinalia.

24. HASSELQUISTIA (egjiptiaca)

Paftinaca orientalis, follis eleganter incifis, Buxb. cent.3.

p. $16 . t .27$, male.

Habitat in Arabia. b.m. Doctor Haffelquift.

Defar. Planta bipedalis. Caulis rigldiufculus villis raris, albis. Umbella compofita, nuda; unlselluthe ravitifiore; involucro polyphyllo, minimo, anguftiffino. Corolla pentapetala, emarginata; Flores in Gifco abostuat. Semina alia orbiculata, glabra, paftinacer majora, in 
medio germine proptio foeta, margine membranaceo pallido cincta, ut in paftinaca genuina. Præterea fune alia Senina hemifperica, glabra, latere hlantia, cava, margine cartilagineo coarctata, marginis bafi adf xa, nec gemina. A Buxbaumio imperfecte picta. Differt ab omnibus duplici \& diverfo fructu, præfertim illo urceolato.

25. SMYRNIUM (egyptiacum) follis floralibus, binis,

- cordatis, integerrimis.

flabitat in Fgypro. D. Hajelquift.

Delcr. Folís infra pedunculos communes bina, oppofita, ovate, amplexicaulia, integerrima. Florés lusei; involicra nulla ; magnitudo vulgaris.

26. PASSERINA (dodecandra) folitis lanceolatis, glabris, acuminatis; floribus lateralibus; ftaminibus quatuor inclufis.

Habitnt in Athiopia. D. Gronovius.

De/er. Planta fere herbacea, Caule lævi glabro, infra folia teret, intra folia tetragono, rarius ramofo: Folia lanceolata, glabra, acuminata, fefitlia, ereêa, longitudine articuiorum. Bractece oppofita sd fingulum forem, tanguam fipule dux lineares, folio breviores. Flos feffilis, tubo longitudine follorum; linbo quadri. partito; Antherce octo, feffiles, barba cincte, tubo impofita, limbo breviores; anthera adhuc quaruor intra faucem tub:.

27. SAXIFRAGA (gcranioides) follis radicalibur reniformibus qutnoelobis inultifits, caulinis linearibus; caule fubnudo ramofo.

Habitnt in Pyreneis, 1hwr/. XVI. 86.

Defor. Folia Ranunculi, reniformia, petiolata, quingueloba, libis fifts. Cnixlis foithamaus, fere nudus, sd ramos foliolotineari. Rami parum diwifi. Flores-misgnt. tudine Saxifraga granulare, calyce in fructum abeunte.

2.8. SAXI. 
28. SAXIFRAGA (ajugrefolia) follis radicalibus palmato quinquepartitis, caulinis linearibus infivifis, caulibus adfcendentibus multifloris. $t$

Habitat in Gallo-Provincie montibus.

Dejer. Folia ad radicem palmata, quinquepartita lobis ovato-lanceolatis, petiolata. Caules adfcendentes, filiformes; follis duobus vel tribus, linearibus, acutis, indivilis. Flores in fummo tres ad quinque, pedunculati. Stolones, antequam folia emittant, hirfuti.

29. GYPSOPHILA (tomentola) foliis lanceolatis fubtrinerviis tomentofis, caule pubefcerte.

Lìnum fylveftre latifolium, flore albicante, Barr, rar: $670, t, 1002$.

Hubitat in Hilpania. Leefling.

Dejcr. Ptanta bipedalis, erecta. Caulis fubvillofus ramis alternis, firmis. Folia lata, Ianceolata, trinervia, obtufiufcula, tomentofa. Flores parvi, albo-purpurafcentes. 3n. DIANTHUS (byflopifolius) follis fubfolitarils, fquamis calycinis longitudine tubi; corollis multifidis; caule erecto.

Caryophyllus fimplex fupinusl latifolius. Bauh. pin. 208? Betonica coronaria feu Caryophyllus minor, follo viridi nigricante, repens, flore argentels punctis notato. Baub. bifl. g.p.32g. fig. mal.

Habitat in Germania, Gallia Narbonenf.

Detcr. Hec planta, quam J. Bauhinus ita fimilem effe Hyfiopo non florente, fi eadem Bauhiniana, non opus habet deferiptione, fed mox ideo dignofitur. Ceulis peralis, erectus. Folia viridia, sec glauca, lariora quam in ieliquis fpeciebus, Petalis multificlis; Calycis lquame fenfim attenuatee, longizudine calycis, quod in nulla alia fpecie multifido flore mihi vifum. Corolle petala 


\section{(4) $) 06$}

purporalcentia, profunde multifida, verfus ungves fupra grifeo barbaza, reflexs. Calyces obfcure purpurafcentes. Corolla magis lacera, \& fere ad báfin, quam in reliquis

31. ARENARLA (miont mia) follis lanceolato-linearibus fcabris, caulibus fterilibus longiflim is procumbentibus. Alfine foliis linearltous acuminatis; pet zlis fiorum int gris, calyce dunlo longioribus. Momi ab/. 127 . Holitat in Gallie aufleclis montihur.

Ditror. Caules cigitales, purpura'cent s. florestes erecti. Steriles rami procumhentes longis flagellis. Folia lanceolata, linearia, acuminata, fcabra, morgine \& carinds magis teabra. Pedunzeuli Tolitarii, folio longiores, uniflori, fubhirti; Calyces acuti. Corolle magne. Petala alha, ovaza, nervola Sicmina \& $P_{1} /$ illa alba.

32. SEDUM r hifpencmin) folis teretiufculis acutis : radtcalibus fafciculatis; cy fa pubefcente.

Sedum bilpanicum, folio glauco acuto, flore albido. Dill. elth. $342 . t .256+332$.

Habitat in Hifpania $\psi$

33. SEMPERVIVUM (birtum) follis caule petalorumque apicibus hirtis,

Sedum majus montanum, folits dentatis. Baub.pin. 283 .

Cotyledon altera montana Cluj. II. bifl.2. P. 63 .

Hibitat in Tauro Raffadienfi.

D.fcr. Folia radicalia acuminata, ciliata. Caulis fublitr. futus , foliis ciliatis villofis. Petala valde longa, apice \& margine fubtus hirra.

34. PRUNUS (infititic) pinofa, folits ovatis lubrus villotis, pedurculis geminis.

Pruna fylveftria precocia. Baub. pin. 444.

Prunus fylveft ris major. Raj. bift. 1528 .

Habitat in Anglia, Germania. Ty/ka Slain.

De/6r. Rami fufca-rubri, leves; ramuli nonnulli /pina 
terminati. Stipule lineares, ciliatæ, acutæ, bafi bifidx, nec ut in dum eftic dimplices \& indivifæ. Eolia ovata, fupra glabra, necut in domeftica fubvillofa, fubtus villora. Gemme fiorifere promunt peduaculos duos ut in domeffica. (ahues glabri, nec villofi ut in domeftica.

35. POTENTILL i (bept phy lhn) folis radicalibus feptenatis cuneiformibus ferratis; caulinis ternatis, raais filifornibus lectmbentibus.

Quinquefolio timilis enneaphyllos hirfuta. Baub. pin. 325 . protix. 13\%

Habitat in Helvetia.

Defer Radix niga, fibrofa, e cujus capiculo fibris nigris foliorum rudimentis obsallato pedunchli plures, digitaJes, viltof, Foliis feptenat s rarius novennatis: Foliolis cuneiformibus, retufis, profunde ferratis lecundum toran fere longitudinem, exterioribus fenfim minoribus, vills vagis albis ailperfis, viridibus nec nttidis: Carles muli, f pithamei, fintormes, virides, villis $\mathrm{ra-}$ ris tongis, bracteis latis bifidis, foliols 3 fepe in uno, feltilibus. Petala emarginata, flava. Piftilla pilofa. Similis $P$. verna, fed Caulis viridis villofior, folia radicalia feptenafa, magis villofa, majora; Caules magis fliformes ; Radix magis nigra.

36. MIMOSA (pigra) foliis bipinnatis oppofize aculeatis: fpina erecta longiore inter fingula partialia, Efchynomere finofa quinta. Comm bort. I p. 59.t.30. Afchynomene folis acacix latioribus, frondibus longisfimos aculeos habentibus. Brevn: cent. $T$.

Hibitat

D./er. Caulis adfperfus aculeis geminis recurvis. Folia fepitus fexjuga: pinnis multijugis. Aculei in petiolis univerfalibus recurvati, inter fingula partialia oppofiti ad latus. At aculeus rectus erectus longior, inter fingu- 
la paria partialium loco glandule. Capitula globofa. Legumina membranaceo-coniprefia, articulata, fcabra.

37. CISTUS (hirta) arborefcens exftipulata, folitis lanceolato-linearibus trinerviis hirtis.

Ciftus ledan hirfutum. Bamb. pin. 467 .

Leson IV. Clufii bift. I. P. 79.t.78.

Habitat Monfpelii $\hbar$

Deler. Rami ruffi, pilis albis ftrigofis adfperfi. Folia lanceolata vel potius linearia, trinervia, margine refiexo, rugofa, urrinque pilis albis nitidis ftrigofis adfperfa, fed fupra minus. Pedunculi longi, villofi, apice aliquor flores fere umbellatos vel alternos preferentes; Calyce ovato, laxo, pentaphyllo; Corclla flava.

38. CISTUS (levipes) fuffruticofus adficendens exftipulatus, folis alternis linearibus glabits, pedunculis racernofis.

Chamrciltus, erice folio, luteus elatior, Ban pin 466. Heliantbemum tenuifolium glabrum erectum, luteo flore, i3aub. bift. 2.p.18

Habitat Monfpeli, b

Delcr. Caulis lignofus, adfcendens, nudus. Folia alterna, remota, linearia, glabra, etjam margine; ex fingulis alis Folia conferta primordia ramorum, fed bre- viora, Racemus eerminalis ramorum laxus, villofovilcidus. Pedicelli remoti, glaberrimi, longi Calyx laviter hifpidus.

Obt. Adeoque hujus fynonyma deleantur in Cilto Fumana. 32. CISTUS (arabicus) fuffruticofus ftipulatus, foliis alternis lanceolatis planis levibus.

Habitat in Arabia D. Hollelquilt. के

Defer. Planta pedalis, fuffruticola, diffufa. Folia lanceola. ta, plana, glabra, acuta; tenella vero fubpubefcentia. Stiputa gentne minimire Pedunculi, ex atis foliorum fuperiorum, folitarii, uniflori. Calyces foliolis tribus 
ovato.cotdatis, acutis, magnis; duobus inferioribus linearibus, scutis, at ntibus. Fructus nutat craffus. 40 CLEMATIS (virginiana) foliis ternatis: foliolis cordatis fcandentibus ferrato-angulatis:

Clematis virginiana pannonicre fimilis, Pluk. mant. 5r. t. 389.7 .4 .

Clematis aquatica trifoliata late fcandens, floribus albis odoratis. Clayt.virg. 62 , n. 270.

Habitat in Penfylvania. $3 i$

Defer. Caules fcandentes, altiflimi. Folia ternata : foliola rarius \& profunde ferrato - angulata, nuda, cordata, venola : Folia floralia fimplicia, fena, aut fubverticillata, integerrima, triioba aut indivifa. Pentunculi fimplices \& triflori, mixti. Corolla tetrapstala, alba, patens, fubtus tomentola : Petalis ovaro-oblongis, patulis, nec lanceolatis. Stemina \& Pifilla albo flavefcentia. Petioluli foliorum compofitorum fe convolvunt $\&$ fcandunt.

41. TEUCRIUM (pumilum) foribus capitatis, foliis linearibus planis quadrifariis confertis, caule procumbente tomentofo. Lafling.

Polium hifpanicum montanum pumilum, rovifnarini folio, Hore rubro. Tourm. inft. $20 \%$.

Polium montanum pumilum rubrum, viridi ftcachadis

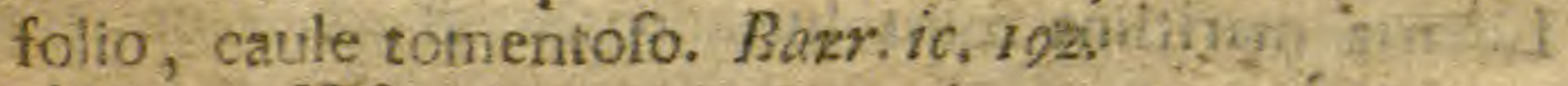
Habitat in Hilpania.

42. OCYMUM (anericanum) foltis fublanceolatis acuminatis ferratis, racemis rotundis, cauld fubliesbadeo.

Habitat in Anterica. Miller. ()

Defor. Canlis erectus, pedalis, ftrifus, tereriufoulus, fcaber, articulatus, Lateribus oppoficés canalicelatés. Renti oppofit, fimplises, brevidres, Gonbulasinter netiolos barbatat Folia oppolisa, petiolata, lancedata feulanceolato-ovata, acuta, nuda, argute fed vix marifolte 


\section{6}

\section{(स) ) 0(}

تe3

nifefte ferrata, bafi canaliculata. Petioli longitudine dimidii folii, pilis longis adfperí. Racemi terminales, folitarii : Verticillis rotundis, fexfloris, remotis, exceptis bracteis duabus, oppofitis, caducis, ovato-lanceolatis, longitudine florum, pilis raris ciliatis. Calyces ante florefcentiam reflexi : Labia fuperiore ovato, bafi gibbo, pilofo: inferiore quadrifido. Corolla alba, calyce duplo major; Stamina ocymi alba, longitudine s) corolke. Stylus incarnatus.

43. ANTIRR HINUM (Jaxatile) foliis lanceolato-linea4. ribus fparfis villofis: inferioribus quaternis; caule decumbente pilofo, floribus fpicatis.

Linaria valentina faxatilis \& perennis villofa, flore luteo. Tourdef. in/t. 160.

Habitat in Hifpania. D. Monnier.

Defor. Radix perennis: Caules decumbentes, villofi, fuperne ramofi. Folia fublinearin, acuta, fparfa, pubefcentia: inferiora quaterna. Spicue terminales. Bractece \& Calyces villuti. Corolla flava.

44. ANTIRRHINUM (molle) foliis quaternis fubulatis, caulibus erectis.

Linaria foliis confertis linearibus carnofis, Hall. gatt 2. $p$. 306 .

Linaria maritima foliis fucculentis. Buxb. cent. 4 p. 23 . t. 37 ?

Habitat in Hifpania. ๑

Defor. Radix annua. Caules fere pedales, erecti, uti tota planta glauco-virides, teretes : Folia fubulata, fubtys planiufcula, fucculenta, erecto-parula; fuperiora alterna, ramea. Spica rarior, terminalis, Calyx patulus apicibus reflexis uti brnftee. Corolle flavæ, ad faucem fere fulvæ, glabræ, labio inferiori divaricato. Calcar lungum, acutum, lineis cærulefcentibus ftriatum. Similis Linarize arvenfi, fed calyx glaber, \& Corolle quadruplo majores.

45. AN- 


\section{(e) ) $\circ(50$}

45. ANTIRRHINUM (Spartum) foliis linearibus alternis, caule paniculato virgato, floribus racemolis, Hobitat in Luifiania, Lafling. (-)

Delor. Caulis fefquipedalis, fullangulatus: ramis teretibus, ramofiffimis. Fotha linearia, longa, fubcarnofa, fupra plana, fubtus rotunda. Calyces racemofi pedicellis letaceis, quinquefidi, acuti, margine membranacei, erecti. Corolle flavie, peralo fulvo glabro; cal car longum, acutum, fiavum.

46. LEPIDIUM (Cardamine) folis radicalibus pinnatis; caulins lyratis Lafting.

Habitat in Hifpanis ad margines viarum argillofas o aridas. Dejor. Folia radicalia omnino Cardamines pinnata, tribus feu quinque foliolis ovatis: extimo rajori. Caules fpithamai, ramofi, terminati racemo. Folia caulina parva, lyrata Flores albi.

47. IBEEIS (baden/is) foliis cordatis : fuperioribus amplexicaulibus. Hill gett.2.p. 243.

Thlafpi alpinum, bellidis cerulex folio. Baub.pih. 106 .

Thlaspi montanum, burfa paftoris fructu. Col. ecph. 1 . p. $275 \cdot t \cdot 276$.

Thlafpi monanum II. Clut. bift. 2. p.13\%.

Habitat in montibus Badenfibus, Aultrix, Matefin Italix, inque Helvetie alprbus. 4

48. IBERIS (arabica) herbacea, folits ovatis glabris aveniis integerrimis, filiculis baft apiceque bllobis.

Thlafpi humile, Ipica purpurea. Buxl. cent. I. p. 2.t 2. f.I. Habitat in Cappadocia , Arabia. Haffelquitt. $\odot$

Defor. Radix fibrofa. Caulis teres, tevvis. Folic ovata, integerrima, acutiufcula, avenia, fucoulenta, glabra. Rami fimplices ex alis foliorum infinis \& furmis, non vero intermediis. Flores coryınbofi parvi rubri; uerum vero petala exteriora majora in fpecimine non vidi, ex facie huc retuli, Silicule magne lato margine mem- 
branaceo, apice fiflo, bafi etiam bilobx. Semina duo in fingulo loculo. 49. IBERIS (p:nnata) folits linearibus pinnatifilis.

Thiafpl umbellatum, nafturiti folio, monfpeliacum. Baub. pin. 106 .

Thlafpi alterum minus umbellatum, nafturtii hortenfis folio, narbonenfe. Lob. ic. 218 .

Habitat in Europa maritimis.

De/cr. Caulis fpithemæus, fuperne ramofus, corymbofus. Folia linearia, pintratifida, glabra. Flores corymbof, petalis duobus exterioribus duplo majoribus. 50. SISYMBRIUM (tenuifolium) foliis integerrimis: infimis tripinatifidis, fupremis integris, Guett. famp.

p. 150. Dalibard parif $204 . t$ Sinapi eruce folio. Baub. pin. gg.

Eruca tenuifolia perennis. Baub. bifl. 2. p.861, Vaill. paril. 50:

H bitat in Gallia, Italia, Helvetia.

Ob1. Cunferantur Planta \& Synonyma cum Sintept fylveftri.

51. SISYMBRIUM (Lcelelir) follis pinnato-haftatis acutis hirtis, caule retrorfum hif ido.

Eryfimum anguititoliura majus. Butb. pin. 107.

Eryfimum hirfutum, filiquits erucer. Laefel. pragl. 60 . t.14.

Eryfimum foliis pinnatis: extrema pinna triangula dentata, petiolis longis hirfutis. Hall. gett.2 p, 248 .

Rapiftrum montanum, irionis folio, microleptoceraton.

Column.cepbr. 1.p. 206. l.208.

Habitnt in Boruflia, Gedani, in montanis equicolor. of 06\%. Adeoque Synonymum Columbe ad Sifymbrium altifimum exchidarur.

52. ERYSIMUM (bierarifolinn) folitis lanceolatis ferra-

tis. Roy. lug 26.34 . Dilith. parf. $20 \%$. Leucojum luteum fylveftre hieracifolium. Baub. pin, zor. 


\section{(e) $) \circ($}

Leucojum fylveftre inodorum, flore parvo pallidiore. Raj, bilt. 78 z.

Habitat in Gallia.

53. CHEIRANTHUS (maritimus) caule ramolo diffufos

foliis lanceolatis, acutiufoulis; antheris eminentibus.

Hefperis maritima fupina exigua. Tourn inft. 223 .

Leucojum maritimum parvum, folio virente craffufculo. Baub. bifl. 2.p. 877 .

Habitat in Europa auftralis maritimis. $\odot$

Defer. Differt a Cheirantho chio in paucis: Caule diffufo. Foliis lanceolatis acutis, vix urrinque unica ferratura notatis, in Chio pluribus. Staminibus \& piftillis extra tubum floris prominentibus.

54. BRASSICA (cbinenfis) foliis ovalibus, fubintegerrimis ; florallbus amplexicaulibus, lanceolatis; calycibus ungvibus petalorum longioribus.

Habitat in China. Dn Dsbeck attulit lemina.

Befcr. Folia oblonga feu ovalia, fimillima Cynoglofio; obtufa fed glabra ; caulina amplexicaulia, oblonga, integerrima. Flores, ut in Braffica oleracea, lutel, Calyx unguibus petalontm brevior, unde hians \& inter peta. la prominens. Stamina longiora. Silique parum compreffer.

55. SINAPIS (orientalis) filiquis retrorfum hifpidis, aptce fubtetragonis, compreflis.

Sinapi orientale maximum, rapi folio. Tourn.cor. I7.

Habitat in Oriente. $\odot$

D./cr. Simillima planta Sinapi arvenfi, fed cota planta retrorfum hifpida; \& filique valuule trifariam retrorfum fcabræ, cum in $S$, arventi glabræ.

56. SINAPIS (incana) filiquis racemo appreftis lævibus, foliis inferioribus lyratis fcabris: fummis lanceolatis, caule fcabro.

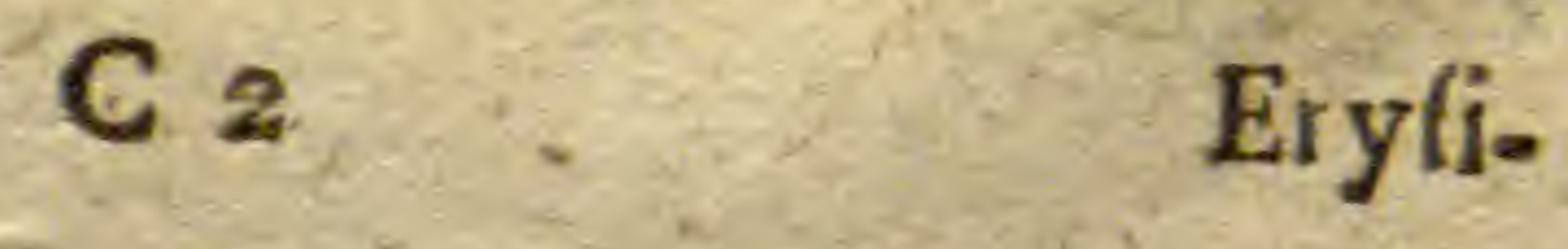


Ery finum follis fubincanis, filiquis breviffimis. Herm.por

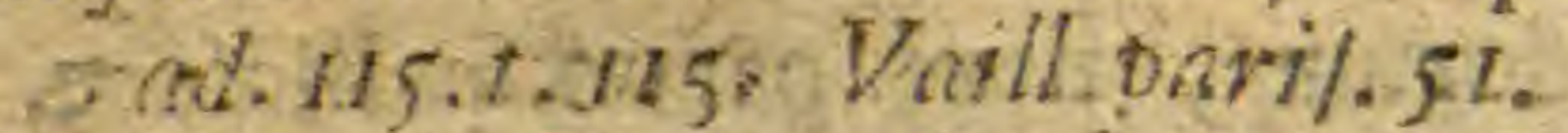
Habitat in Gallia, Libsnia, Hifpania. $\odot$

Defor: Caules triperdales, ramofi, teretes, virides, fcabri. Folia Ganefcentia: inferiora pinnato-iynata, repandc-lentata, utrinque fcabra : extimo ovato majori , caulina fussma, lanceolata, ferrata, petiolata. $R a$ cemi terminales. Flores lutel; Calyces colorati. Petata obtula. Stamina lua as. Silique compreffe, breves, Icapo arcte adpreffe, ut in Eryfino officinali.

57. SINAl1S (levigata) filiquis lovibus patulis, follis lyratis glabris: firmmis lanceolatis, caule lavi.

Eryfimi varieras, Herm parad 155.

Habitat in Lufieania, Hifpania. or 3

DeIcr. Habitus \& omnia antecedentis, fed folia glabra. crulis slaber, laviffmus. Silique fcapo non adpreffe Ted magis diftantes a racemo. Flores majores.

58. CLEOME (arabica), floribus hexandris, foliis finplicibus fubrotundo ovatis, Gliquis gladiatis.

Habitat in Arabia. D. Ha felquift.

D/cr. Planta herbacea, erecta, hifpido-vícida. Folla fubrotundo-ovata, petiolata, integerrima, hifpidovifcida. Racems terminales, folitari, longi. Flores hexandri. Silique latiufcule, compreffe, gladii figura, ad futuram inferiorem magis recurvatæ, fupra bafin glandula notatæ, hifpido-glutinofa.

80. GERANIUM (carno, um) calycibus monophyllis, caule carnofo gibbofo, foliis pinnatifidis, petalis linearibus.

Geranium africanum carnofum, petalis anguftis albicantibus. Dill elth 153.t. $127,7,154$.

Hnbrat in Ethiepia. of

D fir. Differt a Geranio gibbofo Caule carnofo etjam inter at iculos, ubi magis gibbus. Corolle albs, petalis: anguftis .

6. GE. 


\section{(2) 06 (c)}

60. GERANLUM (ciconium) pedunculis fubmultifloris ; calycibus pentaphyllis; foribus pentandrts; folits pinnatis.pinnatifidis obtufis.

Geraniun cicutre follo, acu longiflima. Baub. pin. I32. prodr. 138 .

Geranium apulum coriandrifolium. Col. ecpbr. I. p. 136. t. 135

Hobitat in Europa anftrali, Valefia Italix, Monfpelii, O Defer. Habitus \& facies omnino G anil gruini; fed huic Folia pinnata, decurrentia, pinnaslifida profunde, obtufa, incifa; nec ut gruini zernata, lobata, ferrata. Coulis inclinatus; gruini vero caulefcens eft \& erectus. Petala duo fuperiora magis divaricata ; in gruino æqualia. Glandule in utrisque inter petala.

61. GERANIUM (ver/icalor) pesunculis bifloris, foliis: quinquelobis: lobis medio dilatatis, petalis emarginatis reticulatis.

Geranium romanum verficolor feu ftriatum. Park. par. 229. Morif-biftor. 2. p. $526 \%$. . . t. 16. f. 24. Raj. bift. 1063 . Habitat in Italia.

Dejor. Folia hirfuta, lobis medio dilatatis, incifis, $P e-$ tala alba lineolis purpureo-rubentibus, emarginata. 62. GFRANIUM (diflectum) pedunculis bifloris, foliis quinquepartito-multifidis, caule glabro erectiufculo. Geranium msjus, folis imis longis, ad usque pediculum divifis. Moril. bilt. 2. p. 5 II. J.5. $t \cdot 3 \cdot f \cdot 3$. Vaill paril. $t, 15, f, 2$

Geranitm foliis ad nervum quinquefidis, pediculis brevioribus, caule erecto. Hall. belv. 366 . Habitat in Europa auftraliori.

63. MALVA (totrnefortiona) follis radicalibus quinquepartitis trilnbis, pedunculis folio caulino longioribus, caule fubdecumbente, 
Alcea maritima gatloprovincialis, geranil folio. Iournef.' infl. 98 .

Alcea tenuitolia humilis maritima galloprovincialis, folis inferioribus nonnihil ad geranium accedentibus. Pluk. almi.13.t. 44.5 .4 .

Alcea minor maritima tenuifolia procumbens. Herm. par, 9. t. 9 .

Habitat in Gslloprovincia, Hifpania. Lofling. 50g. a. Defcr. Fulia racticalia petiolata, parva, quinque-vel feptempartita : follolis trilobis, obculis. Caules filiformes, fubdecumbenfou, fcabri, follis duobus vel tribus rantum inftrsear, \& ex fingulis pedunculus folio longior, prater perdunculos plures ad fummitatem plante omnes unittori. Calyx inferior linearis, di vel triplyyllus; interior quinqu filus, latus, fcaber. Differt a Malva mofchata, quod longe tenerior; pecunculis $\mathrm{Al}$ re longiontbus, folins minimis, in caule tantum duobus vel rribus minus pinnatifidis. Figura Herbianni \& Plukenetii ex planta culta, folia nimis acuta pingunt.

64. GENISTA (ciandicons) folitis ternatis, fubtus villofis; pedunculis Jateralibus lubquinquefloris foliatis; leguminibus hirfutis.

Cytifus monfpeffulana, medicx folio, filiguis denfe congeftis \& villofis. Toumef. in/t. 648 .

Cytifus fylveftris candicans Calalp.plant.113, Habitat Monfpelii $\varepsilon^{\circ}$ in Italia. D. Snawougés.

Defor Rami angulati, villofr. Folia brevibus petiolis ternata, ovalia, utrinque acurta, fubtus multum villofa, fupra minus. Ex atis fuperiorum foliorum peduculi erecti, fere ramei, apice duobus vel tribus foltis minoribus inftructi, intra quos Flores quinque vel feptem in fafcienlum collecti. Calyces flavefount. Corollde Geniltze tinctorix. L'egumina valde hirfuta. 
65. LUPINUS (Aoloniferus) calycibus alternis appendicuJatis: labio fuperiure bipartito, inferiore tridentato, leguminibus lanatis.

Lupinus lanuginofis latifolius humilis, flore caruleopurpuraicente, Atoloniferus. Cup. Catbol.

Habitat in Sicilia, Archipelago, Arabia.

Delcr, Planta humilis, vix fpithamrea. Folie quinata, longe petiolata. Foliola bafin verfus anguftiora, lanceolara, pilis teftaceis feu ferrugineis adfperfa. Flowes alterni, carulefcentes. Legumina veftta pilis longis, deniis, ferrugineis, tosde tota planta ferrughes evadit. C6. PHASEOLUS (nonus) caule erectiofculorrvi, leguminibus pendulis compreflis rugofis. Hort, $1 \mathrm{p} / .213$. Phafeolus vulgaris italicus hurntlis fou minor, albus cum orbita nigricante. Bucub. bift 2. p.35\& R ni. bift. 885 . Smilax filiqua furfum aigente, feu Phafeolus parvus ita. licus. Baub.pin.339,

Habitat in India.

Def $c_{0}$ Variat flore albo \& purpureo; Semine albo \& fufco maculis lateis. Flos duplo minor, quam in vulgari. 6\%. OROBUS (/yluaticus) caulibus decumbentibus hirfueis ramofis. $t$

Orobus fylvaticses noftras. Raj. angl.g. p. 921 . /uppl. bift. 1892. Adt. Paril.1706. p. S7. 4.98.

Hubitat in Anglia, Gallia 4

Deler. Caules ramoli, Ariati, decumbentes. Eoliola fexaut fepremjugata. Corolle extus rubentes, intus albre lineis purpureis. Legumina compreffa. Semia duo vel tria.

68. CORONILLA (glaica) fruticofa procumbens, foliolis obcorlatis, leguminibus teretibus. Salibaget.

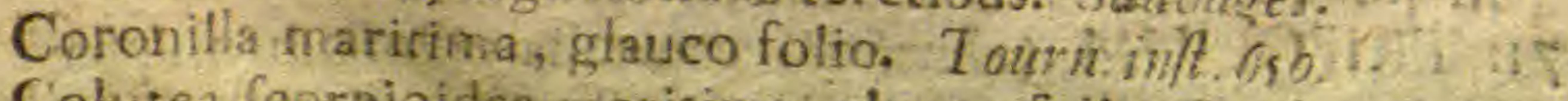
Cohtea forphoides matizima, glauco folio. Lalub pin. 397 . prodi. 157. 
Habitat in Gallia Narbonenfi.

Obf- Diltinct a Coronilla valentina caulibus non erectis, nee leguminibus articulatis.

- 69 . TRIFOLIUM (Arictum) capitulis globofis, legumini1 bus difpernter caule erecto, folis lanceolatis ferrulatis, fipulis rhermbeis. $t$

Trifolium pratenfe annuum erectum minimum, folis long angutis pulchre venatis \& tenuifime ferratis, flo.

- ribus alble In captetum congeftis, tiliquis ninoribus difpernife. Micb.zen. 20.t.25.t.7.

Habitat in Irslla pratis, patcuis. (a)

70. TRIFOLIZM (uniflorum) pedunculis communibus fubtifforis, Anpulis foliorum brevioribus.

Trifolium vernum repens purpureum. Buxb. cent.3.p. 17.t.31. $f .1$.

B Trifolium vernum repens, flore albo exiguo. Buxb. cent. 3 p. 17.t.31.f. 2.

Habitat Conftankinopoli, inque Syria, Arabia, Juexa, Hajpelquif.

- Confer. Melilotus cretica humillima humifufa, flore albo magno. Tourn.cor. 28.

Spica trifolia. Alp. exot, 160 2.169.

Defer. Coules procumbentes, terre adprefli, undique veftivi flipulis foliorum rumidis, apice bifílis, e quibus $F_{0}$ ha petiolis long sernata, fervulata. Pedunculi ex fingulis vaginis conimunes folitaril, brevifini, terminati pedicellis duobus vel tribus, fipulis paullo longioribus, (unde flores quafi folicarii,) quibus tingulls infidet fos fimplex, nec capitulum, unde pedunculi communes bi-aut triflori. Calyres nan hirti, fed oblongi, in quinque fegmenta acuminata terminati.

71. TRIFOLIUM (cherlexi) capiculis villofis hemifphæricis terminalibus folitariis, calycibus omnibus tertilibus. 


\section{(c) $) 01$}

Trifolium capitulis villofis globolis terminalibus bractez írbiculata terminatis. Saw mon/p. 184 .

Habitat in Hilpania. ?

Defer. Diverfa planta a Trifolio globofo. Caules fimplices, breves, adfcendentes, pilofi ut tota planta. Capitulo in fingulo ramo unico, feffili, terminali, ho mihbarico, exceptum Atipulis latiffimis orbiculum conftituentibus, tanquam caíyce capitulum amplectentibus \& excipientibus, quarum altera foliolis tribus fesfilibus, altesa nüllo gaudet. Flo/culi omnes fertiles. Calyces fetaceil, villofi, chrolla longiores, adfperf pilis albis nitidiffinis, unde fingularis calycis facies.

Objerv. Adeoque fynonyma Trifolii globofi Sp. Pl, 768. ad hanc revocantur, \& in eor tale capite lanugino/o. Tourn cor. 27. quod habitat in Arabia \& Syria.

72. TRIFOLIUM (alexendrinum) capitulis pedunculatis, folis infimis oppofitis.

Habitat in Fypto. D. Hagelquift.

De/er. Planta vix pedalis, erecte. Cmulis inferne nudus, in medio Folia duo oppofita, reinata: Aipritis lanceolatis, temibifidis, attenuatis. Petiolt foliolis paullo breviores, extrorfun fefé latiores. Folvola la to-lanceolata, vix manifelte crenata. Pentunculos ex altera ala, nulus, longus. Capitulum oblongum, album. Cautis ex altera ala continuat, red folis alternis, ceterum fimilis. Galgees parum villofi. Habirus Trifo. lii montani.

73. HYPERICUM (10abrum) floribus trigynis; caule tereti, fuffruticofo, muricato; folits oblongis.

Hypericum orientale, caule alpero purpureo. Tourn.torilg. Habitat in Arabia. D. Haljelquift. क

$D$ ofr. Caules fruticofi, fenefcentes cortice deciduo rugofi, herbacei, rufefcentes, ad!perfi punctis elevatis, 
unde fcabri evadunt. Folia oppofita, ovali-lanceolata, Invia, integerrima. Corymbi terminales, floribus parvis rnigynis. Caulis punctis eminentibus ut in ba. learico, led longloribus, folia autem absque glandulis mihi manifeftis, \& flores omnino trigyni diftingvunt a balearico.

74 HYPERICUM (repens) floribus trigynis; caule tere. ti repente; foliis lanceolato-linearibus, obtufis. Hypericum orientale, polygoni folio. Iourn. cor.19. Habitat in Palæettina \& adjacentibus. D. Huffelquilt. Defor. Radix perennis \& Itgnofa fupra radicem. Caules teretes, filiformes, repentes, ad genicula radiculas dimittentes, Folia lanceolato-linearia, obtufa, integerrima, confertim fatis oppofite. Flores terminales tres, intermedio feffili. Calyx \& bractee punctis fufcis ad margines adfperfi, non vero ferrati.

75. LEONTODON (lanatum) foliis enfiformibus, integris, hirfutis; calyce erecto, fimplici.

Chondrilla altera. Raunolf. itin. 2i7. t. 217.

Chondrilla bulbofa fyriaca, foliis latioribus, Baub.pin. 131 . Habitat in Arabia. D. Haffelquilt.

Defor. Radix bulbola. Folia lanceolato-linearia, plurima, digitalia, usrinque lana alba hirfuta, radicalia. Scapi foliis paullo longiores, aphylit, in medio fepe unico foliolo lineari minimo notati, hirfuti. Flores magnitudine Pilofellæ; calyse fimplici, rquali, polyphyllo, longitudine fere corolle, lanato. Corolla lutea. 76. HIERACIUM (tomentofum) foliis oblongo-ovatis, hirfutis, fefflibus, fubdentatis; pedunculis multifloris. Hieracium montanum tomentofum. Dill, eltb.181. $t$. 150. f. 180.

Habitat in Europa auftrali. $\psi$

Defer. Planta perialis, tota villis incanis hirfuta, ramofa, Folia ovato-oblonga, obfolete dentata. Rami multiflori, Calyces hirfuti. 77. EU. 
(e) $) \circ($

27

7\%. EUPATORIUM (maculatum) folis quinis, Innceo. Iatis, æqualiter ferratis, petiolatis, venofis.

Delcr. Folia quinque ad genicula, lanceolata, xqualiter ferrata. Caulis tenuifime maculatus. Varietes Euppato. rii purpursi ad hoc, ut \& ejus fynonyma \& defcriptio Ppectant. Eupatorium enim purpuretum follis quaternis, lanceolato-ovatis, inæqualiter ferratis, rugofis eft.

78. ARTEMISIA (nilotica) foliis bipinnatis; caule eretiufculo ; pedunculis folitarils, unifloris, nudis, filt? formibus.

Habitat in IEgypto. D. HaJelquift.

Deler. Radix fibrofa. Caules pedales, herbacei, ere: ctiufouli, fubvillofi. Folic pinnata, pinnis pinaatifidis, facie Artemifie annuxe. Flores globofi, magnitudine \& figura annua, fed pedunculi ex axillis foliorum, filiformes, aphylli, longitudine fere foliorum, us niffori.

79. GNAPHALIUM (/angvineum) foliis decurrentibus, lanceolatis, utrinque tomentofis, planis, apiculo nudo terminatis.

Gnaphalium ægyptiacum, latiore flore ramofius, flore ex albicante purpureo. Pluk. mant $9 I$.

Gnaphalio montano affinis agyptiaca. Baub. pin. 264.

Chryfocoma fyriaca, flore atro rubente. Breyn. cent. 46 . Raocharis diofcoridis. Ranwolf. itin. 285.t. 285 .

Habitat in Palæeftina, Agypto. D. Hajelquift.

De/cr. Caulis pedalis, non ramofus, teres, villofus. Folia lanceolata, decurrentia, utrinque tomentofa, apiculo vix confpicuo rufo terminata, prefertim inferiora. Corynbi terminales, faturate rubri, magnitudine \& $\mathrm{co}$ lore Gnaphalii dioici ; ftatura arenarii.

80. BACCHARIS (diofcoridis) folits lato-lanceolatis, dentatis, feffilibus, ftipulatis.

Conyza major altera. Baub. pin. 265.

D 2

Cony- 
Conyza diofcoridis. Rawwolf.itin. 54.t.54.

Habitut in Syria, IEgypto. D. Haflelguift.

Detcr. Coulis erectus, purpurafcens, ftriatus. Folia alcerna, lato-lanceolara, feflilia, \& fere femi-amplexicaulia, ferrato-dentata, fupra nuda, fubtus vix manifette villofa. Rami ex alis fuperioribus, caule altiores, erecti; Pedunculi terminales, corymbofi : foliolis minimis, linearibus, integerrimis, acutis ad divaricationes Calyces fubcylindriei. Corolle luteæ.

81. ERIGERON (graveolens) ramis lateralibus multifloris, calycibus fquarrofis. Sauvages.

Virga aurea minor, folis glutinofis \& graveolentibus. Ioumet. inft. 484 .

Conyza femina Theophrafti, minor Diofcoridis. Baub. pin. $26 \%$.

Conyza mings vera, Lobel. ic. 346 .

Habicat Monfpelii. O

Defer. Eft Evigero vifcato decies minor. Folin integerri-

ma. Rami nulbifori. Flores pallide lutei.

82 VERPESINA (nodiflara) follis opnolitis; calycibus coblongis , filibus, confertis; caule taterali

Bidens nodiflora, folie tetrahit Dill.etib.53.t. 45.7 .53 .

Chryfantheraum conyzoides norliflorum, femine roltra.

to bidente. Slogn tams 126.bif. I. pag.262.t.154, H, Halitat in Caribeis. O

Obferv. Semina radil ina ginibus lateralibus dentato-ciliata funt

83. HELIANTHUS (trondo/us) ealyolbus fquarrofis; untdularis, fron ofis; radiis octopetalis.

Habitot in Canada,

D for. Statura Hetianthi decapetali, a quo differt corollis triplog najoribus, fere Helianthi multiflori; at radit vix vitra cto funt. Flores erects funt. Caulis quadripedalis, folitarius. Folia argute ferrata, lata, Pe. dincu- 


\section{(ien) $) \circ(\operatorname{ces})$}

dunculi foabri. Folia calycina divaricata, acuta, ciliata, hifpida, undulatim flexa, \&-infima /quama $\mathrm{fx}_{3}$ pe in folium enata. Sic differt ab. Heliantho giganted caule duplo breviore. Ab Heliantho decapetalo coral la duplo majore. Ab Heliantho multifloro radiis pau cioribus \& calyce lquarrofo.

84. COREOPSIS (leucanthema) foliis pinnatis fetratis, radio divérficolore.

Bidens americana trifolia, leucantheml flore. Lournefort. inft. 462 .

Habitat in America. Miller. 0

De/cr. Caulis eriun vel quatuor pedum, tetragonus, leviufculus : lateribus oppofitis canaliculatis. Rami oppofiti. Folia oppofite, pinata : Foliolis quinque, rarius tribus, ovatis, indivifis, glabris, aqualibus, argute ferratis, levibus, extimis tribus fxpe confluentibus, latere exteriore fere decurrentibus, apice integerrimis. Petioli canaliculati, utrinque villo connexi. Flores terminales, alterni, duo vel tres, pedtucults longiufculis. Caljx quinquepartitus, involucro feu exteriore calyce pentaphyllo minore. Corolle radius fapius pentapetalis, albus. Petalis ovatis, apice tridentatis, intermedio broductiore; fubtus trinerviis. Difcus paryus, convexus, luteus. Semina corona tridentata, retrorfum hifpida; feminum difcus pyramtdalis evadit. Planta Bidentis frondoli. Flos Afteris annui. 85. CENT AUREA (calcitrupoides) calycibus fubduplicato-fpinofis, foliis aniplexicautibus lanceolatis ferratis. Carduus ttellatus, folis integris ferratis. Magn. monlp. 202.

Habitat Monfpelii, $\varepsilon^{\circ}$ in Palaftina. D. Hallelqui/t.

Defer. Tota planta Centaurea Calcitt pa, fed coulis \& if folia thinus hirfuta, \& folia minime divifa, teriuigtme ferrata, acuminata, lanceolata, antplexicaulia. $\mathrm{Ca}$ - 
- lyces axlllares, feffiles, omnino Calcitrapo, fed ad bafin lanuginofi. An planta hybrida?

86. CENTAUREA (Verutum) calycibus fimpliciffime fpinofis : dentibus duobus oppofitis, foliis lanceolatis integris decurrentibus.

Habitat in Palxftina. 0 D. Haffelquill.

De/cr. Radix fibrofa. Caulis pedalis, fimplex, angula: tus, erectus. Rami breviores, fimplices, ex alis fuperioribus. Folia lanceolata, integerrima, fcabra, decurrentia. Flores terminales, folitarii. Calyces fubtomentofi. Spinis fimplicibus, flavis, ftriatis, calyce duplo longioribus, in eo fingulares, quod quevis fpina gerat in medio duas oppofitiones ex fpinis minimis, erectis nec patulis: ad bafin vero fpine flavefcentes absque ferraturis aut aculeis, Corolle flava.

87. CENTAUREA (prmilks) calycibus fimpliciffime (pinofis, folits dentato-pinnasis villofis, caule nullo.

Carduus orientalis acaulos incanus \& tomentofus, dentis leonis folio. Tournef. cor. $3 T$.

Habitat in Epypto. D. Halfelquift.

Defor. Simillima Centaurea acault, fed calycis /quame non ciliatz. Radix dura. Calyces frepius tres, radici arcte adnati, ovato-oblongi, glabri : fquamis margine membranaceis, integris, aplce ovatis, \& in /pinam fubulatam, brevem, flavefcentem, terminatis; Corol. luta oblonga. Flores neutri vix reliquis longiores. Fo. lia linearia, dentato-pinnata : dentibus ovato-lanceolatis, latioribus fuo folio, alternis fape parvis; omnia cinerea fubvillofa, radici incumbentia.

88. SERIPHIUM (gnapbaloides) floribus fpicatis unifloris.

Habitat in Æthiopia. D. Royen.

Defcr. Rami angufti. Folic linearia, fparfa, tomentolas, fupra nuda. Flores in Jpicam congefti, flavi, triftes, fubto- 
fubtomentofi, cylindracei, continentes flofeulum unum, cujus corolle lacinie acute reflexæ, \& quafi un. cinatee.

89. CALLITRICHE (androgyina) foliis fuperioribus avalibus.!

Corifpermum folis oppofitis. Fl. Lapp. 2. Fl. Sv. 3 Hort. Clif. 3. Gronov. virg. 3. Roy. lugdbat. 205.

Stellaria aquatica. Baub. pin. 14 I.

Stellaria follis omnibus fubrotundis. Hall. gatt. 2. p.39. Habitat in Europa folis, vere florens. 90. CALLITRICHE (bermapbroditica) foliis omnibus linearibus, apice bifidls.

Stellaria foliis omnibus anguftis, apice refeeto. Hall. belvet. 198.

Lenticula paluftris anguftifolia, folio in apice diffecto. Loefel.prut].140.t.138.

Habitat in Europe fofis, autumno florens.

$\mathrm{Ob}$. Excludatur itaque Callitriche paluftris.

91. CAREX (brizoides) fpica compolita, difticha, nuda; fpiculis androgysis, oblongis, contiguis, patentibus; culmo nudo.

Carex fpicis teretibus, acutis, alternis, fe contingentibus. Hall. belv. 244 .

Carex fibrata radice, anguftifolia; caule exquifite trian. gulari. Micb. gen. 70 t. 33 f. 17 .

Graroen cyperoides elegans, fpica compolita afperiore. Raj.bilt. 1297. Morif. bill.3.p 245.

Habitat in Europa.

92 CAREX (remota) fpicis ovatis, fubfeffilibus, remo- tis, androgynis; foliis caulinis culmum xquaptibus. $t$ Carex fpicis in foliorum alis feffilibus. Guetiard. ftamp. I. 1. 1$)^{2} t$. Dalibard parif. 151 .

Cyperoides anguftifolium, fpicis feffilibus in foliorum alis. 
alis. Scherez gram. 483. Raj hift. I295. Morif. hilt.3. $\$$. 243. 1. 8.t.12.t 17. Pluk.alm.178.t.34.f.3.

Habitat in Europæe umbro/is lubbumidis. 4

Obl. Spice valèe parvæ; inferiores fæpe pedunculo brevi. 93. CAREX (piniculata) racemo compofito.

Carex fpica paniculata. Hell. belo. 246.

Cyperus longus inodorus fylvaticus. Baub.pin. I 4.tbeatr.22I.

Cyperus alpinus longus inodorus, panicula ferruginea minus fparfa. Scbeuchz. prodr.7.t.8.

Gramen cypeiroides paluftre, iplca longiore laxa. Raj.bift. 1296. Mor\%.bift.3.p:044.1.8.t.12.f.23. Scbeusbz gram. 499. Habitat in Europx auftralioribus utigino/is. 24

94. AMARANTHUS (polygamus) fpicis diandris, ovatis, feflilibus, axillaribus ; foliis lanceolatis acutis.

Hubitat in India. (o)

Defer. Conlis erectus, fefquipedalis, viridis, ftriatus, lavis. Fulia lanceolsa, petiolata, nuda, petiolis fub bafi tumidiufculis; ex axillis ramus \& copitulum, quod conftat pedunculo breviffimo, multifloro utrinque ad bafin ramo connivente in capituium viride. Flores diandri. Spica terminalis, brevis, ovata, parva, tere uti capitula lateralia. Fl. hermaphrodili diandri digyni,

95. AMARANTHUS (mangoftanus) fpieis triandris, glomeratis, feffilibus, axillaribus, terminalibusque ; foliis rhombeis obtufis.

Habitat in India. (o)

Dejor. Caules pedales \& ultra. Flores glomerati, virides, ariftati, ad axillas \& in Spicam interruptam, cerminalem. Foliz rhombea, obtufffima, latiora quam longa, viridia, bafi cuneiformia, longis petiolis infidentia, longioribus ipfo folio, folia apice fape parum emarginata. 96. THUJA (apbvlla) ftrobilis quadrivalvibus : tolis turbinatis vaginantibus, hine mucronatis, frondibus im. bricatis. 


\section{(4) $) 068$}

Cupreflus fructu quadrivalvi, folits equifeti inftar articus latis. Sbaw. afric. 188

Habitat in Egypto. क

De/cr. Habitus Tamaricis; Frondes filiformes, laxi. Rams articulatim cincti vaginis minimis, foliaceis, truncatis altero latere, apiculo inftructis, quæe vaginacea folia in tenellis frondibus imbricata, ut quafi aphylia confpiciatur. FruCtum non vidi.

27. CUCUMIS (propbetarum) foliis cordatis, quinquelobis, denticulatis, obtufis; pomis globolis, fpinofomuricatis.

Colocynthis pumila echinata arabica, ftriis duodecim lo. teis \& viridibus variegata. Sbaw. afric. 164 .

Habitat in Arabia. D. Halfelquift.

Defor. Flanta habitu Melonis, fed parva. Folia ribefik cordaza, obtula, profunde triloba ultra medium, finu obtufo: lobis laseralibus minus profunde bilobis; margine dentato, feabro, venis fubtus magis hifpidis. Calllis repens feu fcandens, quinquangularis. Pormum fubglobofum, undique prinis diftantibus muricatum. 28. SALIX (egyptiaca) foliis fubferratis, lanceolato-ova: libus, nudis, venofis; petiolis fimplicibus exftipa-

Salix fyriaca, folio bleagineo argenteo. Bmib.pin 474.

Calaf leu Ban, Alp. rgypt. 6r. z.62.

Habitat in Rigypto, D. Halfelquill. क

Delcr. Rami craffi, rubri, toeves. Folia alterna, figura S. capreee, ovalia, acutiufcula, utrinque nuda, fubtus venis inæqualia, vix manifefte \& remote ferrata, petiolis brevibus infidentia. Gemma magnitudine faba, purpurea, univalvis. Amentum lanatum, figura Salicis caprex, flo/culis diandris.

99. VALANTIA (cucullaris) fructificationibus fingulis bractea ovata deflexa tectis, 
Cucullaria. Buxb. cent. I. p.13.t.10:, .2.

Hobitat in Cappadocia \& Arabiæ montefis. $\odot$

Defcr. Pisuta palmaris. Caulis tetragonus, erectus, ramofus. Folic quaterna, lanceolata, ad flores vero oppofita Pedunculi ramofi, multiflori, ex fummis alis. Supra fingulum flofculum Bractect ovata, membranacea, colorata, vexillum floris papilionacei referens, occultans fernen \& fiorem.

Ico. A TRIPLEX (glauca) caule fuffruticofo, procumbente; folis ovatis, feffilibus, integerrimis: inferioribus fubdenratis.

Atriplex maritima hifpanica frutefcens \& procumbens. Dill. eltb. $40 . t \cdot 40 . f \cdot 46$.

Polygonum incanum rotundifolium halimoides fruticans: hilpanicum. Barr. rar. $1455^{t}, 733$.

Habitat in Gallia auftrali of Hifpania maritimis. th. IOI. A TRIPLEX (pedunculata) caule herbaceo, divaricato; foliis lanceolatis, obtufis, integris; calycibus: femineis pedunculatis.

Atriplex maritima, femine lato. Raj.angl' 3.p.153.

Atriplex maritima Halimus dicta, humilis, erecta; fol.

liculis in fatitidinem expanfis \& utrinque recurvis, longo pediculo infidentibus. Plink. $a \ln , 61 . t \cdot 36 \% \cdot \mathrm{t}$ Habitat in Anglixe moritimis, Hafnix. Tych. Holm. 0 Difor. Rami divaricati. Racemi ramos terminant, \& ex alis exeunt, pedunculis lateralibus, firplicibus, binatis feu Aggregatis, quorum apici infidet Calyx turfe paftoris iructui fimilis, trilobus, intermedio minori.

\section{$A D D E N D A$ :}

17. A dimirabilis Jafmin roffa. Clut, bift 2. $p$ go. 18. Atzoyatl Mirabilis mexicana. Hernand mex: $170, f, 2$. 


\section{(ए) $)$ o \\ THESES. \\ है: \\ 35}

\section{THESIS $\mathrm{s}$.}

Hifforia Naturalis recte difingui debet a PhiTofopbia Naturali.

\section{THESIS 2.}

Peregrinationes multum fecum, tam Medickne, quam Oeconomite, utrlitatis adferre; nemo inficias ire poteft.

\section{THESIS. 3.}

Medicamenta fimplicia, inprimis beroica, $a b$ exteris $\mathcal{G}^{\circ}$ barbaris nationibus, multo cum

- fructu ufitais, in noftra Pbarmacopolos introduci debent.

\section{THESIS 4 :}

Planta officinales a que nofurum clima ferunt, excoli $\mathcal{E}^{2}$ pußunt $\mathcal{E}^{\sigma}$ debent.
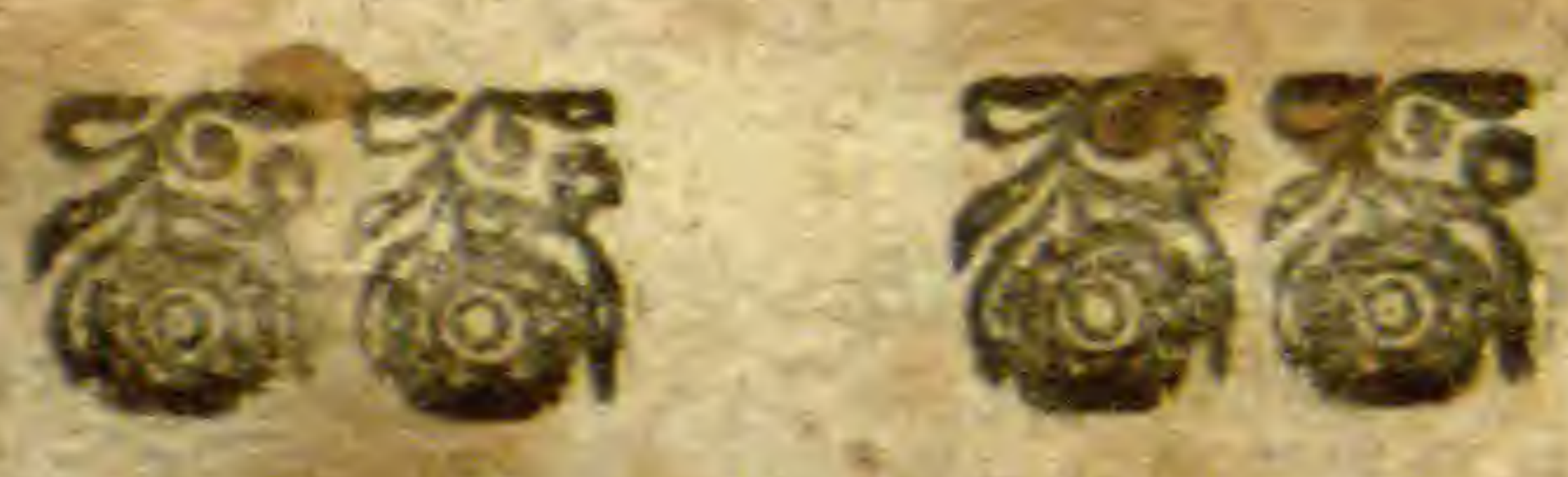

t 


\section{$F R A T R I$ fivo}

Cariffimo.

Cpecimine præfenti, quo, Frater dilectis$S$ fime, Centuriam plantarum nuperrime detectarum cum kepublica communicas Litteraria, quid merueris, melius norunt jucundiffinæ pariter ae utiliffmæ Botanices periti, quam quidem ego, qui vix delicias ejus, primis, quod ajunt, degu. Atavi labris. Novi faltem, quod opus fic utile, \& parum non faciar ad incremen. ta Hiftorix Naturalis, Gratulor igitur tibi, Frater dilectiffime, hanc occafionem tuam oftendendi induftriam, \& quemad. modum spero, te non inglorium ex arena difputatoria difceffurum, ita $\mathrm{ex}$ intimis cordis receffibus voveo, velit DEus T.Q.M, conatibus tuis ita adlpirare, ut tandem metam, quam tibi propoluifti feliciffime attingas, meflemque, laboris tructum, reportes uberrimam. Sic folatiùm Matri, jam jam ad lenium vergenti, Cariffmre, \&c gaudium adferes eis, qui tibi langvinis vinculo conjuncti funt, \& amicis omnibus. Vale.

Scare d. IX. Kalend, Fan. Ann. MDCCLV.

ERICUS JUSLENIUS: 